DR M. SANTER (Orcid ID : 0000-0001-7264-5260)

Received Date : 28-Nov-2016

Revised Date : 03-Feb-2017

Accepted Date : 14-Feb-2017

Article type : Original Article

\title{
Views of oral antibiotics and advice seeking about acne: a qualitative study of online discussion forums
}

Running head: Online discussion forums for acne

M. Santer ${ }^{1}$, D. Chandler ${ }^{1}$, M. Lown ${ }^{1}$, N.A. Francis ${ }^{2}$, I. Muller ${ }^{1}$

1. University of Southampton

2. Cardiff University

Corresponding author:

Miriam Santer

Primary Care and Population Sciences

University of Southampton

Aldermoor Health Centre

Aldermoor Close

Southampton SO16 5ST

02380241019

m.santer@soton.ac.uk

Funding sources: none

Conflict of interest: none declared

This article has been accepted for publication and undergone full peer review but has not been through the copyediting, typesetting, pagination and proofreading process, which may lead to differences between this version and the Version of Record. Please cite this article as doi: $10.1111 /$ bjd. 15398

This article is protected by copyright. All rights reserved. 


\section{What's already known about this topic?}

- $\quad$ Acne vulgaris is very common and can have considerable impact on quality of life.

- $\quad$ Although some information is available about patients' views of oral isotretinoin, little is known about views and experiences of the most common treatments for acne, including oral antibiotics.

- $\quad$ Online discussion forums are an increasingly common source of information about health conditions, yet we know little about the advice exchanged about acne online.

\section{What does this study add?}

- We found very diverse views about oral treatments in general and oral antibiotics in particular.

- Sources of frustration included the delayed onset of action of antibiotics for acne, perceptions of only temporary effectiveness and adverse effects.

- $\quad$ Advice on some discussion threads seemed inappropriate because the severity of acne was not taken into account; advising sunshine for severe acne or dermatology referral for 'some spots' for example.

\section{What are the clinical implications of the work?}

- $\quad$ People seeking information about acne in online forums are likely to find bewildering advice with potentially negative as well as positive impacts.

- $\quad$ Clinicians prescribing oral treatments for acne need to provide information and support around the delayed onset of action.

- $\quad$ Health professionals can help avoid confusion by signposting people with acne towards reliable, evidence-based resources to access outside the consultation.

\section{ABSTRACT}

\section{Background}

Acne vulgaris is common and can significantly impair quality of life, yet little is known about patients' understanding of acne and its treatments. Oral antibiotics are widely used for acne, despite concerns about antibiotic resistance. People are increasingly turning to online discussion forums for advice and information on these sites may influence health beliefs and treatment adherence.

\section{Objective}

To explore understandings about the use of oral antibiotics for acne and advice shared amongst messages posted on online forums.

This article is protected by copyright. All rights reserved. 


\section{Methods}

We systematically searched for online forums and identified four where acne was frequently discussed. Discussion threads relating to oral antibiotics were analysed thematically. NVivo 11 facilitated data handling.

\section{Results}

We extracted 136 pages of data; 65 discussions amongst 294 participants. We found a wide range of perceptions around effectiveness of antibiotics for acne and concerns about adverse effects. The delayed onset of action of antibiotics was a source of frustration and compounded dissatisfaction with healthcare providers, who people perceived as 'fobbing them off' with prolonged courses of ineffective treatment. Advice ranged from costly cleansers to when to ask for, or insist on, referral. Posts related to a wide range of severities, from 'spots' to severe acne, which may make it confusing for users to assess appropriateness of information.

\section{Conclusions}

Online forums offer opinions that could be confusing or lead to early abandonment of treatments, challenging consultations and patient dissatisfaction. Users expressed frustration about the delayed onset of action of antibiotics for acne, perceptions of only temporary effectiveness and adverse effects.

\section{BACKGROUND}

Acne vulgaris is a very common condition. Some degree of acne affects almost all teenagers and is moderate to severe in 15 to $20 \%$, often persisting to adulthood. ${ }^{1}$ It is estimated that $30 \%$ of teenagers have acne that may require medical treatment. ${ }^{2}$ The main impact of acne is psychological, with increased risk of depression and suicide ${ }^{1,2}$ and psychosocial disability similar to that seen in conditions such as asthma and diabetes. ${ }^{2}$

Over $3 \%$ of people aged 13 to 25 years consult for acne in primary care in the UK each year. ${ }^{3}$ Guidelines suggest step-wise treatment involving (i) single agent topical, (ii) combination topical, (iii) oral antibiotics plus topical treatment. ${ }^{2}$ However, oral antibiotics are widely prescribed for acne in the UK, ${ }^{4}$ despite concerns about the development of antibiotic resistance. ${ }^{5,6}$ It is not clear whether this over-use of oral antibiotics is due to patient pressure or doctor preference.

Qualitative research has explored the impact on adults and young people of living with acne ${ }^{7,8}$ and on people's views of oral isotretinoin ${ }^{9}$, yet little is known about people's views of other acne treatments. This matters because health beliefs have a strong influence on treatment adherence, ${ }^{10}$ which is a common barrier to effective treatment in acne. ${ }^{1}$

This article is protected by copyright. All rights reserved. 
In $2015,78 \%$ of adults in Great Britain used the internet every day or almost every day. ${ }^{11}$ Almost $50 \%$ of adults currently use the internet to access health information, a figure which continues to rise. ${ }^{11}$ Online forums are discussion sites that allow users to communicate their personal experiences through asynchronous written communication and are increasingly being used to seek information from others with similar health issues. ${ }^{12}$ Online discussion forums are often more popular than static websites because people value first-person accounts and feel supported by others in the online community. ${ }^{12}$ Online forums provide qualitative researchers with a rich and valuable source of data about users' perspectives and experiences and there has been an increase in qualitative research exploring online exchanges relating to a range of health-related conditions. ${ }^{13-15}$

Online forums appear to impact both positively and negatively on health behaviours, for instance through helping people find information and feel supported, influencing behaviour (through reading others' experiences) and informing how to navigate health services (including asking questions of health professionals). ${ }^{12}$ Exchanges in online forums generally have a permanence, providing searchable information available to future forum users with a potential for impact on many users. ${ }^{16}$ A recent BMJ editorial commented that, 'The conversations patients have in their online communities must be brought closer to the conversations currently conducted among health professionals. ${ }^{17}$ One way of working towards this is for health professionals to gain a better understanding of the concerns and views shared in online communities.

Qualitative exploration of discussions about acne in online forums can allow insight into forum users' viewpoints and an appreciation of advice that they may have received prior to consulting. This study focusses on perceptions of oral antibiotics as these are widely used for acne and there are concerns about the impact of this on rising antibiotic resistance. ${ }^{5}$ We therefore aimed to investigate understandings and concerns about the use of oral antibiotics for acne and explore the nature of advice seeking for acne amongst people who have posted messages on online forums.

\section{METHODS}

This qualitative study sought to explore messages (and their resultant discussions) about oral antibiotics posted by people with acne, and their carers, in UK-based discussion forums.

\section{Data retrieval}

Initial scoping review of online resources was carried out in June-July 2016 by entering the terms 'acne', 'acne antibiotics', 'teenage acne antibiotics' and 'acne forum' into three different commonlyused search engines, using the 'UK results only' option, and looking at the first 100 hits. We found discussion of oral antibiotics for acne within acne-specific forums, forums for other medical conditions and general medical forums, parenting forums, and general discussion forums. Only active forums considered to be in the public domain were included in this study. Forums were excluded if they required registration to view posts, contained fewer than three relevant threads or had not had any posts within the last six months. There were 12 remaining forums, of which we selected four in order to yield a manageable amount of data. We chose four forums offering diverse perspectives, choosing one acne-specific forum, one general forum, one general medical forum and

This article is protected by copyright. All rights reserved. 
one parenting-focused forum, selecting the one with the greatest of number of acne-related posts from each category. All four listed a UK address.

Due to variation in the availability and capabilities of the internal search functions of the selected forums, a uniform search strategy could not be applied. The researcher (DC) familiarised himself with each forum to tailor an appropriate search strategy (Box 1).

Posts were defined as relevant if they included views or experiences of oral antibiotics, or were part of a conversation that included these. Threads were initially only included if they had been posted during or after 2010, but within these threads all relevant posts were included regardless of date, in order to retain context for the later posts. In July 2016, 90 pages of data were collected from the four selected forums, but review of these suggested that more data were needed to saturate the main themes. An additional 46 pages were therefore collected in early August 2016 (Figure 1).

Once extracted, threads were anonymised and unique identifiers were assigned to each thread and to each username. The anonymised data were transferred to NVivo11 to facilitate data handling.

\section{Data analysis}

An inductive thematic analysis ${ }^{18}$ identified common themes running through the discussions. Coding began following reading and re-reading of the data. A sample of the discussions were coded line by line (DC) in order to produce an initial coding frame. This was discussed amongst the research team, initial themes and subthemes were identified and a draft coding manual was produced. This manual was iteratively developed (DC, IM, ML, MS), and then used to interrogate the data for themes and subthemes and to search for disconfirming cases. As analysis progressed it became clear that considering threads in the context of the initial post was necessary to contextualise comments and data was re-read and coded in this way.

\section{Ethical issues}

Approval was granted by University of Southampton Faculty of Medicine Ethics Committee (reference 22431). This study considers online forum messages that can be viewed without registration to be located within the public domain, in line with guidelines from the British Psychological Society ${ }^{19}$ and in line with the approach used in other studies. ${ }^{13-15}$ Discussions posted in the online forums examined for this study are accessible by anyone and so seeking informed consent is not applicable. Steps have been taken to maximise the anonymity of participants: forums are not identified by name, thread titles and usernames have been replaced with unique identifiers and quotes have been paraphrased to further protect participants' identities. All authors agreed the adapted paraphrased quotations prior to submission.

This article is protected by copyright. All rights reserved. 


\section{RESULTS}

Forum searches yielded 136 pages of text comprising 65 discussions containing posts relating to the use of oral antibiotics for acne (Table 1). These discussions dated from 2007 to 2016 and appeared to have been posted by 294 different users, although it is possible that some users used more than one username. The discussions were generally supportive, sometimes conversational, often taking the form that one user started a thread with a question or request for advice, followed by a number of responses. The acne-specific forum included posts from users who appeared to be based outside the UK (as judged from their comments), whereas the other forums mainly appeared to be used by UK residents. The acne-specific forum contained more posts that appeared to come from individuals with commercial interests, for instance directing others towards private nutritionists. The parenting forum appeared to have greater site moderation (i.e. an employee or forum user who responded to some of the comments or removed spam), who sometimes directed thread-starters to relevant pages on NHS Choices.

Inductive thematic analysis of data from the four forums revealed themes relating to antibiotics and other treatments, navigation of services and the impact of living with acne. This paper will focus on themes relating to views and concerns about oral antibiotics (effectiveness, adverse effects and frustrations around delayed onset of action) and the nature of the advice on offer in the discussion forums.

\section{Perceptions around effectiveness and appropriateness of oral antibiotics for acne}

Online forum users expressed a range of views relating to the use of oral treatments in general and oral antibiotics in particular. Reasons given for not wanting to use oral treatments included a general preference for 'natural' remedies, whereas others felt that 'treatment from the inside' was essential for successful treatment of acne, or that topical treatments were "mild" while oral treatments were "strong".

I'm constantly taking my daughter to the doctors, as her acne has spread so much. We even saw someone at the hospital, but nothing we have been given so far has been any help. They said she might end up on tablets, but I don't like the sound of that - she's only a kid! Before she tries that I'm going to look in [cosmetics shop] and see what they have.

Parenting forum user193

I gave benzoyl peroxide a go, but I don't think it does any good to treat acne on the outside unless you fight it on the inside as well.

General forum user79

Many users reported success with antibiotics but many also reported that they received temporary success with oral antibiotics, but that symptoms reappeared or worsened when they stopped. There was a general perception of oral antibiotics becoming ineffective, often presented in terms of the person themselves becoming 'resistant' or 'immune' to the medication.

This article is protected by copyright. All rights reserved. 
Some users were posting within days of starting treatment with oral antibiotics, seeking advice about how long they would take to work. A few users, rather than expressing a belief that antibiotics either would or wouldn't help, simply expressed their hope or desperation, while a few seemed confused about whether their oral treatment was an antibiotic or not.

I went to the doctor because my skin was upsetting me so much. I'm just praying the antibiotics they've given me will fix it.

General medical forum user37

I hope the roaccutane antibiotic they've given me will make things better.

General forum user112

While many forum users regarded oral antibiotics as effective, or partially effective, there were also many who perceived them as ineffective. Oral antibiotics were sometimes only mentioned briefly amid wider posts listing treatments that had been tried prior to eventual resolution of acne with oral isotretinoin. The latter was often contrasted with antibiotics (and other treatments) as a highly effective and long-lasting treatment, although there was widespread awareness of common side effects (e.g. dry skin/lips) and concerns about possible mental health side effects.

After several appointments with the doctor my son has ended up on a topical lotion and some antibiotics, but I know they won't do any good. I think roaccutane would be much better for him, so I'm taking him to see a dermatologist privately.

Parenting forum user140

\section{Adverse effects with antibiotics}

Perceived negative effects associated with taking oral antibiotics were commonly reported, sometimes lasting beyond cessation of treatment, particularly gastro-intestinal side effects or 'weakened immune system'. There were some report of people stopping their antibiotics due to the negative effects they associated with them, mainly nausea or other gastrointestinal side effects.

The tetracyclines I tried did help quite a bit in the end, but taking them was so terrible and it was taking so long to get better that I gave up on them. One gave me an itchy rash, while the other made me feel really sick.

Acne forum user8

The parenting forum included a thread of discussion around the negative effects of taking lymecycline while pregnant, in which several users mentioned struggling to access detailed information on the impact this would have on their unborn child.

This article is protected by copyright. All rights reserved. 


\section{Delay in onset of action of oral antibiotics}

People frequently discussed the delay in onset of action of oral antibiotics and that waiting for effect was a difficult time. Many seemed to have been advised to expect this delay, although a few users appeared to think they would act within days.

Well, 4 days in and still not looking any better...

Parenting forum user150

The delay in onset of action was a source of frustration to many ("I hate all this waiting"), particularly those who were desperate to see improvement in their acne. Some perceived the delay in onset of action and need for several months' use in order to judge effectiveness as part of being "fobbed off" by their GP. People who wished for oral isotretinoin as their treatment of choice felt that being asked to try antibiotics for some months first was an unwelcome delay and this was a source of dissatisfaction with healthcare providers in some instances.

I'm on antibiotics too. My doctor said I have to take then full course before I can try something else. Three months is so long to wait - I feel like I'll be dealing with this forever.

General forum user61

Antibiotics don't do anything for acne, the doctor just gives you them to fob you off.

General forum user281

\section{Variable advice and experiences in acne severity}

As with many online discussion forums, the quality of the advice users offered each other was highly variable but, added to that, people appear to be discussing very different sorts of 'acne', varying from 'some spots' to severe nodulocystic acne. Many users appeared to assume that they were talking to someone with acne similar to their own, for instance, people with experience of severe acne sometimes advised others that they must see a Dermatologist regardless of severity.

My skin is so bad and nothing I try helps - I've tried different skincare routines, my diet is healthy and I drink plenty of water. It's just gets me down to see everyone with great skin around me when mine looks like this.

General forum user277

You should DEFINATELY go to a doctor, and be adamant about a referral to dermatology. I have been seeing a dermatologist and they are really helping. Don't wait, as your skin will get worse if you leave it.

This article is protected by copyright. All rights reserved. 
Others were advising cosmetic or lifestyle advice where advice to consult seemed more appropriate.

Well, I'm spending yet another hot sunny day sitting in my bedroom. I have nodular acneworse than the normal teenage thing. I can't show my face outside when I look like this. I used to be so happy, and now I think about killing myself. I haven't seen some of my friends in at least a year. I have tried everything to treat it but nothing helps.

General forum user284

You could give Neem oil or Tea Tree Oil a go.

General forum user285

It has major side effects, but as your acne is affecting you so much you should consider Roaccutane. You would need a dermatology referral from your GP to get it.

General forum user286

Sunlight is great for the skin, so you should go out as much as you can!

General forum user287

Go see a doctor?

General forum user288

Many people strongly recommended that people who were suffering from acne seek help from a doctor, either for medication the GP could prescribe, or for a referral to dermatology. It was striking how frequently users were urged to pressurise their GP for a referral to a Dermatologist. This may be related to input from people with a history of severe acne, or because of input from users based outside the UK (particularly in the acne-specific forum). Sometimes people advised seeking a dermatology referral for treatments that a GP can prescribe, or advised people with relatively mild skin problems to seek referral, which may have led to difficult consultations.

Only one user commented that no-one knew each other's severity and reflected that their doctor might be well-placed to judge acne severity.

There's loads of information available on the internet... but ultimately only your doctor will really be able to decide how severe your acne is and whether it is getting better. No-one here can help you with that. It'd be a really good idea to go back to see your GP...

General forum user275

This article is protected by copyright. All rights reserved. 


\section{DISCUSSION}

We found a broad range of views and experiences relating to oral antibiotics expressed in online discussion forums and a mix of potentially helpful as well as unhelpful advice. Reported experiences with oral antibiotics ranged from rapid and total success to making the acne worse and causing intolerable side effects. As such, it is difficult to predict what attitude may be fostered in forum users who seek advice about oral antibiotics, other than a likelihood that they would find this a bewildering experience. Dissatisfaction with oral antibiotics related to a variety of factors: a perception that the antibiotics didn't work or stopped working with time, experiences of side effects, and a general reluctance to take antibiotics.

\section{Findings in the context of existing literature}

Forum users referred to antibiotic resistance both in relation to both topical and oral antibiotics but, as has been found in other studies, ${ }^{20}$ antibiotic resistance was generally interpreted as a property of the human body rather than bacterial cells. 'Resistance' was generally only mentioned as a reason for treatment to stop working; neither the long term implications for the user nor the wider implications of overuse of antibiotics for healthcare were discussed, though it is unclear whether this is because of a lack of awareness or because it wasn't relevant to the discussion thread. This is concordant with findings from other studies, which have found that participants often view antibiotic resistance as being of low risk to themselves and more a topic for concern for clinicians or other people. ${ }^{21}$

We found that forum users frequently discussed experiences with consulting doctors, generally frustration, and often advised each other to pressurise GPs for referral to a Dermatologist. This echoes qualitative research carried out amongst patients with other dermatological conditions suggesting that they may face particular challenges in the doctor-patient relationship; including feelings of 'not being taken seriously' and that both GPs and Dermatologists can be perceived as not engaging with the psychological distress associated with skin disease. ${ }^{22-24}$ Qualitative research on eczema has highlighted a 'mismatch' of views between patients/carers and health professionals that can add to frustrations. ${ }^{25,26}$

The finding that people often obtain advice online about how to navigate health services, as well as the potential for both positive and negative impacts (in this case, both useful advice and potentially confusing or inappropriate advice) have been found in other studies of online discussion forums. ${ }^{12}$

\section{Limitations}

This research has limitations, some of which are inherent to carrying out qualitative research in online discussion forums. While this allows access to rich naturalistic data, little information available about participants' demographics, which precludes purposive sampling. This presents a particular difficulty with acne, which is so commonly experienced and so varied in severity. Contextual information on severity and impact would be highly relevant to understanding attitudes to treatments. However, to find themes in common within this heterogeneous sample suggests that

This article is protected by copyright. All rights reserved. 
these are highly relevant to understanding the concerns of people with acne. It may be that people who use online discussion forums differ in important ways from those who do not and it would be useful to carry out further research to explore this.

\section{Research implications}

Although we have found signals pointing towards explaining the popularity of oral antibiotics (e.g. the perception that topicals are 'mild' and oral treatments are 'strong'), further exploration of overuse of antibiotics for acne could focus on qualitative interviews with both health professionals and people with a range of acne severities and treatment experiences. In some non-dermatological conditions it is thought that patients place pressure on health professionals to prescribe antibiotics due to a perception that they form a 'magic bullet', ${ }^{27}$ yet we do not know whether this is the case in acne.

Further research is needed to find effective ways of communicating with people with acne (for instance through online resources), including providing support during delayed onset of action of treatments, support with adherence and addressing questions arising outside the consultation.

\section{Clinical implications}

These findings can help clinicians to understand the perspectives of patients with acne, and gain an insight into the highly variable information that they may access prior to consulting. Assessing information needs and health beliefs is an important part of the dermatology consultation ${ }^{28}$ and a key role of health professionals is to signpost people towards high quality evidence-based information free from commercial bias, ${ }^{1}$ particularly as it is known that patients do not recall a substantial proportion of information given to them during the consultation. ${ }^{29}$ A quantitative study of online forum posts for Acne rosacea found extensive information needs and the authors concluded that if doctors offered patients reliable sources of information then this may prevent patients treatment non-adherence and following potentially erroneous advice from the internet. ${ }^{30}$

\section{CONCLUSIONS}

This study shows that online forums present readers with diverse advice and views on oral antibiotics, which could at best be confusing and at worst lead to early abandonment of treatments, requests for inappropriate referrals, challenging consultations and patient dissatisfaction. Some of the more potentially helpful posts directed readers towards reliable sources of information, such as NHS Choices. If patients with acne were directed to this information then they may experience better treatment outcomes.

This article is protected by copyright. All rights reserved. 
$1 \quad$ Williams HC, Dellavalle RP, Garner S. Acne vulgaris. The Lancet 2012; 379: 361-72.

$2 \quad$ Acne vulgaris. In: Clinical Knowledge Summaries - CKS. 2014.

3 Purdy S, Langston J, Tait L. Presentation and management of acne in primary care: a retrospective cohort study. British Journal of General Practice 2003; 53: 5.

4 Francis NA, Entwistle K, Santer M et al. The Management of Acne Vulgaris in Primary Care: A cohort study of consulting and prescribing patterns using CPRD. British Journal of Dermatology 2016: n/a-n/a.

5 Walsh TR, Efthimiou J, Dréno B. Systematic review of antibiotic resistance in acne: an increasing topical and oral threat. The Lancet Infectious Diseases 2016; 16: e23-e33.

6 Eady AE, Cove JH, Layton AM. Is Antibiotic Resistance in Cutaneous Propionibacteria Clinically Relevant? American journal of clinical dermatology 2003; 4: 813-31.

7 Murray CD, Rhodes K. 'Nobody likes damaged goods': the experience of adult visible acne. British journal of health psychology 2005; 10: 183-202.

8 Prior J, Khadaroo A. 'I sort of balance it out'. Living with facial acne in emerging adulthood. Journal of Health Psychology 2015; 20: 1154-65.

9 Magin $\mathrm{P}$, Adams J, Heading $\mathrm{G}$ et al. Patients' perceptions of isotretinoin, depression and suicide--a qualitative study. Aust Fam Physician 2005; 34: 795-7.

10 Horne R, Weinman J. Predicting treatment adherence. Adherence to Treatment in Medical Conditions, edited by Lynn Myers and Kenny Midence 1998: 25-50.

11 Office for National Statistics. Internet Access - Households and Individuals In. http://www.ons.gov.uk/peoplepopulationandcommunity/householdcharacteristics/homeint ernetandsocialmediausage/bulletins/internetaccesshouseholdsandindividuals/2015-08-06 (accessed 15 June 2016). 2015.

12 Ziebland S, Wyke S. Health and Illness in a ConnectedWorld: How Might Sharing Experiences on the Internet Affect People's Health? The Millbank Quarterly 2012; 90: 41.

13 Attard A, Coulson NS. A thematic analysis of patient communication in Parkinson's disease online support group discussion forums. Computers in Human Behavior 2012; 28: 500-6.

14 Coulson NS. Sharing, supporting and sobriety: a qualitative analysis of messages posted to alcohol-related online discussion forums in the United Kingdom. Journal of Substance Use 2014; 19: 176-80.

15 Teasdale E, Muller I, Santer M. Carers' views of topical-corticosteroid use in childhood eczema: a qualitative study of online discussion forums. British Journal of Dermatology 2016: n/a-n/a.

16 Allen C, Vassilev I, Kennedy A et al. Long-Term Condition Self-Management Support in Online Communities: A Meta-Synthesis of Qualitative Papers. Journal of Medical Internet Research 2016; 18: e61.

17 Richards T, Snow R, Schroter S. Co-creating health: more than a dream. Bmj 2016; 354.

18 Braun V, Clarke V. Using thematic analysis in psychology. Qualitative Research in Psychology 2006; 3: 77-101.

19 Hewson CBT BI, Coulson NS, et al. . The British Psychological Society: Ethics Guidelines for Internet-mediated Research. In. 2013.

20 Brookes-Howell L, Elwyn G, Hood K et al. 'The Body Gets Used to Them': Patients' Interpretations of Antibiotic Resistance and the Implications for Containment Strategies. Journal of General Internal Medicine 2012; 27: 766-72.

21 McCullough A, Parekh S, Rathbone J et al. A systematic review of the public's knowledge and beliefs about antibiotic resistance. Journal of Antimicrobial Chemotherapy 2016; 71: 27-33.

22 Magin PJ, Adams J, Heading GS et al. Patients with skin disease and their relationships with their doctors: a qualitative study of patients with acne, psoriasis and eczema. Med J Aust 2009; 190: 62-4.

This article is protected by copyright. All rights reserved. 
23 Nelson PA, Chew-Graham CA, Griffiths CE et al. Recognition of need in health care consultations: a qualitative study of people with psoriasis. The British journal of dermatology 2013; 168: 354-61.

24 Gore C, Johnson RJ, Caress AL et al. The information needs and preferred roles in treatment decision-making of parents caring for infants with atopic dermatitis: a qualitative study. Allergy 2005; 60: 938-43.

25 Santer $\mathrm{M}$, Burgess $\mathrm{H}$, Yardley L et al. Experiences of carers managing childhood eczema and their views on its treatment: a qualitative study. The British journal of general practice : the journal of the Royal College of General Practitioners 2012; 62: e261-7.

26 Noerreslet M, Jemec GB, Traulsen JM. Involuntary autonomy: patients' perceptions of physicians, conventional medicines and risks in the management of atopic dermatitis. Social Science \& Medicine.69(9):1409-15 2009.

27 Pinder R, Sallis A, Berry D et al. Behaviour change and antibiotic prescribing in healthcare settings. Literature review and behavioural analysis. In: 2015. London: Public Health England.

28 Hong J, Nguyen TV, Prose NS. Compassionate care: enhancing physician-patient communication and education in dermatology: Part II: Patient education. J Am Acad Dermatol 2013; 68: 364 e1-10.

29 Ley P. Memory for medical information. British Journal of Social and Clinical Psychology 1979; 18: 245-55.

30 Alinia H, Moradi Tuchayi S, Farhangian ME et al. Rosacea patients seeking advice: Qualitative analysis of patients' posts on a rosacea support forum. Journal of Dermatological Treatment 2016; 27: 99-102.

This article is protected by copyright. All rights reserved. 


\section{Figure 1}

Online forum search strategy

Forums identified through search engines: $n=33$

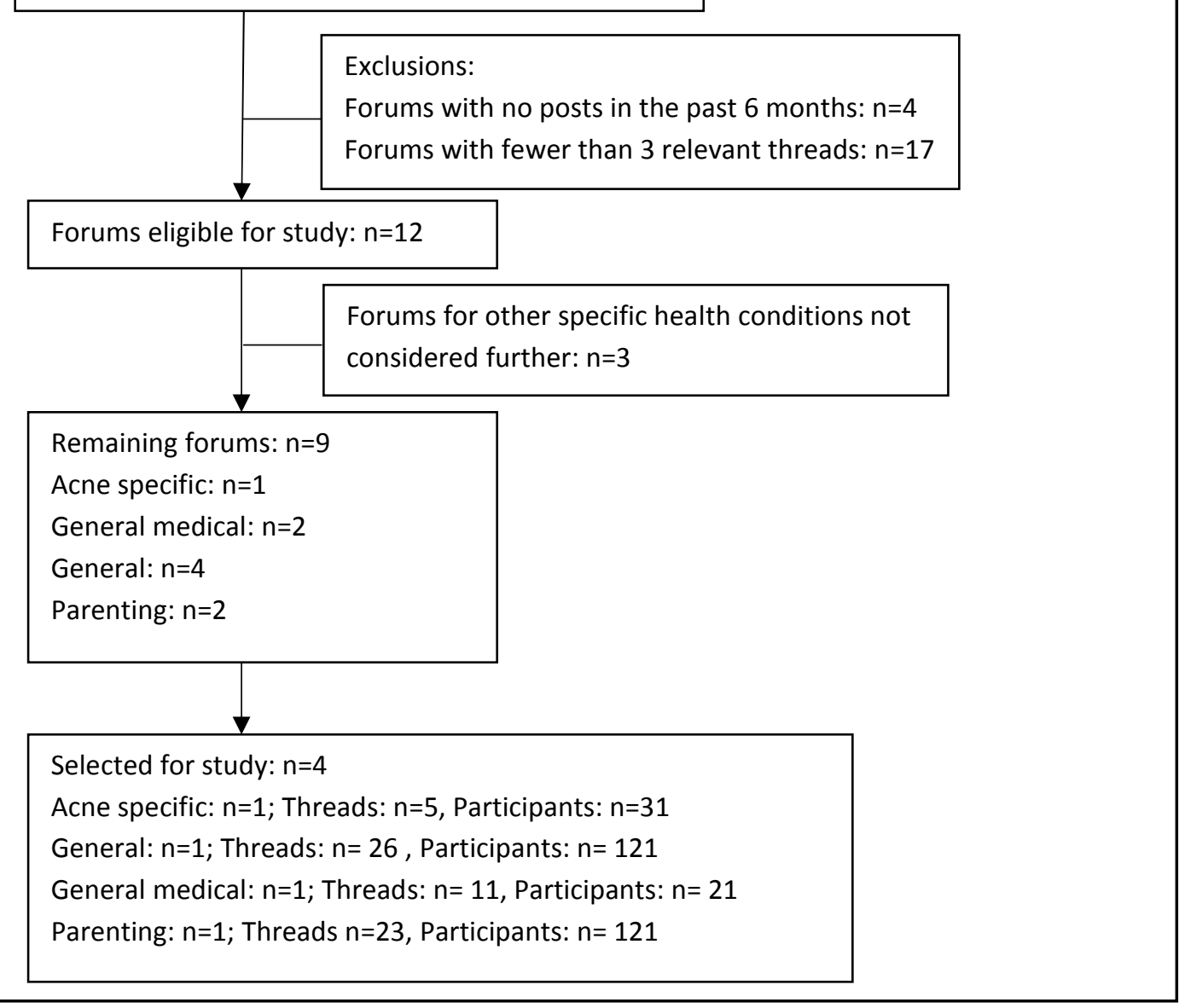

This article is protected by copyright. All rights reserved. 


\section{Box 1}

- For the acne specific forum, where no viable search engine was present, the forums were searched manually for relevant threads until a sufficient volume of data was obtained or all relevant threads had been extracted.

- For the general and the general medical forums, where a chronological search function was present, the researcher searched for "acne antibiotics", then "acne", then "acne treatment". In each case, relevant threads were identified from newest to oldest, and relevant posts extracted. Posts were extracted from the general forum until a sufficient volume of data was obtained; the general medical forum had fewer relevant threads, so all relevant threads were extracted.

- For the parenting forum, where a search function was present but results were not returned chronologically, the researcher searched for the terms described above, and then the first 10 pages of each search were examined for relevant threads. Once all relevant threads were identified, these were examined chronologically, from newest to oldest, and relevant posts extracted until a sufficient volume of data was obtained.

Table 1

\section{Data sources}

\begin{tabular}{|l|l|l|l|l|}
\hline & $\begin{array}{l}\text { Number of } \\
\text { pages of text } \\
\text { (point 11) }\end{array}$ & $\begin{array}{l}\text { Number of } \\
\text { relevant } \\
\text { discussion } \\
\text { threads }\end{array}$ & $\begin{array}{l}\text { Number of forum } \\
\text { users contributing } \\
\text { to threads }\end{array}$ & $\begin{array}{l}\text { Dates of } \\
\text { discussion } \\
\text { threads }\end{array}$ \\
\hline $\begin{array}{l}\text { Acne-specific } \\
\text { forum }\end{array}$ & 16 & 5 & 31 & $2009-2016$ \\
\hline General forum & 57 & 26 & 121 & $2007-2016$ \\
\hline $\begin{array}{l}\text { General medical } \\
\text { forum }\end{array}$ & 9 & 11 & 21 & $2008-2015$ \\
\hline Parenting forum & 54 & 23 & 121 & $2011-2016$ \\
\hline $\begin{array}{l}\text { All 4 discussion } \\
\text { forums }\end{array}$ & $\mathbf{1 3 6}$ & $\mathbf{6 5}$ & $\mathbf{2 9 4}$ & $\mathbf{2 0 0 7 - 2 0 1 6}$ \\
\hline
\end{tabular}

This article is protected by copyright. All rights reserved. 